Published in final edited form as:

Trends Genet. 2011 August ; 27(8): 307-315. doi:10.1016/j.tig.2011.05.004.

\title{
Centrosomes and Cilia in Human Disease
}

\author{
Mónica Bettencourt-Dias ${ }^{1}$, Friedhelm Hildebrandt ${ }^{2}$, David Pellman ${ }^{3}$, Geoff Woods $^{4}$, and \\ Susana Godinho ${ }^{3}$ \\ ${ }^{1}$ Instituto Gulbenkian de Ciência, Oeiras, Portugal \\ ${ }^{2}$ Howard Hughes Medical Institute and Departments of Pediatrics and of Human Genetics, \\ University of Michigan, Ann Arbor, Michigan 48109, USA \\ ${ }^{3}$ Howard Hughes Medical Institute, Department of Pediatric Oncology, Dana-Farber Cancer \\ Institute, Children's Hospital, Harvard Medical School, Boston, Massachusetts 02115, USA \\ ${ }^{4}$ Department of Medical Genetics, Cambridge Institute for Medical Research, University of \\ Cambridge, Cambridge, UK
}

\begin{abstract}
Centrioles are microtubule-derived structures that are essential to form centrosomes, cilia and flagella. The centrosome is the major microtubule organiser in animal cells, participating in a variety of processes from cell polarization to cell division, while cilia and flagella contribute to several mechanisms in eukaryotic cells from motility to sensing. Although it was suggested more than a century ago that these microtubule-derived structures are involved in human disease, the molecular bases of this association have only recently been discovered. Surprisingly, there is very little overlap between the genes affected in the different diseases, suggesting there are tissuespecific requirements for these microtubule-derived structures. Knowledge of these requirements and disease mechanisms has opened new avenues for therapeutical strategies. Here, we give an overview of recent developments in this field focusing on cancer, diseases of brain development and ciliopathies.
\end{abstract}

\section{Centrioles, centrosomes and cilia}

The centriole is a conserved eukaryotic organelle involved in a variety of processes, such as cell division and motility (Fig. 1). This structure, in addition to other proteins, is made primarily of microtubules organized most often in nine triplets (Fig. 1A, $\mathrm{A}^{\prime}$; reviewed in ${ }^{1}$ ). The centriole participates in the formation of the centrosome, the major microtubule organizing center (MTOC) in animal cells (Fig. 1A), which coordinates cell division, motility and polarity. The centrosome is comprised of two distinct centrioles surrounded by an electron-dense matrix, the pericentriolar material (PCM). While the PCM harbours molecules that anchor and nucleate cytoplasmic microtubules (MTs) in interphase and mitosis, centriolar characteristics determine most properties of the centrosome, such as stability and capacity to reproduce (reviewed in ${ }^{1}$ ). The centriole also sets up the foundations

(C) 2011 Elsevier Ltd. All rights reserved.

Authors for correspondence: Mónica Bettencourt-Dias- mdias@igc.gulbenkian.pt; Susana, Godinhosusana_godinho@dfci.harvard.edu.

Publisher's Disclaimer: This is a PDF file of an unedited manuscript that has been accepted for publication. As a service to our customers we are providing this early version of the manuscript. The manuscript will undergo copyediting, typesetting, and review of the resulting proof before it is published in its final citable form. Please note that during the production process errors may be discovered which could affect the content, and all legal disclaimers that apply to the journal pertain. 
for the axoneme, the skeleton of cilia and flagella that are structures involved in sensing and movement (Fig.1B).

The number of centrioles in a cycling cell is normally controlled through a duplication cycle (Fig. 2A). During the cell cycle, new centrioles form orthogonally to the two existing ones, giving rise to two centrosomes. This occurs in coordination with DNA synthesis in S phase. Thus, when the cell enters mitosis it is equipped with two centrosomes, each harbouring two centrioles, which nucleate and anchor microtubules that form the mitotic spindle (Fig. 2A). As a consequence of the centrosome cycle, a dividing cell harbours 3 centriole generations, which have different ability to nucleate microtubules and form cilia. Non-random patterns of inheritance of differently-aged centrioles have been observed in stem cells where centriole age seems to play a role in asymmetric cell division ${ }^{2-5}$.

Not every cell follows the centrosome cycle. Some cells have many centrioles and others have none. Many ciliated cells, such as those in vertebrate respiratory and reproductive tracts, can have 200-300 cilia per cell. This requires the generation of multiple centrioles, each forming one cilium. Both in multi-ciliated cells and in cells without pre-existing centrioles, centrioles are formed de novo (reviewed in ${ }^{6}$ ). Centrioles are universally required for the assembly of cilia, but that is not the case for cell division. Several cell types divide without centrioles, the classical examples being higher plants and oocytes (reviewed in ${ }^{7}$ ). However, other cells depend on these structures for accurate cell division, such as embryos and spermatocytes from a variety of species, suggesting that centrioles might have been coopted for cell division in certain tissues ${ }^{8}$ (discussed in ${ }^{7}$ ).

Cilia can be motile, in which case they are sometimes also called flagella, such as in the sperm, or immotile, such as primary cilia that exist in most of our cells. Both types of cilia have sensory functions, with some being specialised in that function, such as photoreceptors ${ }^{9-11}$. In recent years an interplay between several signalling pathways and primary cilia has been shown ${ }^{9-11}$ (Fig. 2B). For example, the absence of cilia leads to many Hedgehog related phenotypes, as part of the signaling occurs in this structure. The binding of Hedgehog ligand to Patched-1 leads to Smoothened translocation to the ciliary membrane, activation of the Gli1 and Gli2 transcription factors and Hh pathway activation (reviewed in ${ }^{10}$ ). Primary cilia have been proposed to play a role in Wnt signaling pathways (reviewed in ${ }^{12}$ ). Wnt can act via the canonical pathway through Dishevelled, by repressing the degradation of $\beta$-catenin and promoting proliferation and differentiation. Wnt can also act through non-canonical pathways, such as the planar cell polarity (PCP) pathway, which regulates the cytoskeleton and is important to organize cells in the plane of the epithelium, through the orientation of cell divisions. The role of cilia in Wnt signaling is controversial (for an extensive discussion please refer to ${ }^{12}$ ), it is however clear that proteins involved in PCP can affect ciliogenesis, in particular basal body docking and orientation ${ }^{12}$. Finally, localization of the PDGF receptor-alpha to cilia is important for cell migration mediated through this signaling pathway (reviewed in ${ }^{10}$ ).

Theodor Boveri first established a link between centrosome abnormalities and disease more than 100 years ago by proposing that an abnormal increase in centrosome number could lead to abnormal mitotic spindles, hence be a source of chromosome instability that could cause cancer ${ }^{13}$. Other roles of centrioles and centrosomes in human disease have mostly been driven by the discovery in recent years that centrosomal genes are associated with a variety of human diseases, such as ciliopathies and diseases of brain development. Here, we discuss the very recent findings of the role of centrosomes and centrioles in cancer, brain development and ciliopathies and what they tell us about the functions of those organelles in tissue development and homeostasis. 


\section{Centrosome abnormalities in tumors}

While most of the cancer field has focused on the understanding of specific gene malfunctions that lead to cancer, Boveri's early predictions only recently became an area of active investigation. The idea that centrosome abnormalities play a role in tumorigenesis was revived by the observation that knockdown of the tumor suppressor p53 leads to centrosome amplification in mouse fibroblasts and in skin tumors ${ }^{14}$. It was also established that centrosome abnormalities are common in a variety of human tumors, such as breast, prostate, lung, colon and brain ${ }^{15,16}$. Extensive analysis of a variety of solid tumors and hematological malignancies has further reinforced these observations ${ }^{17-19}$.

Generally, centrosome defects can be classified as structural or numerical aberrations ${ }^{20}$. Structural defects, likely a result of altered expression of centrosomal proteins, can lead to abnormally enlarged centrosomes and enhancement or reduction of MT nucleation. Numerical abnormalities, such as centrosome amplification, are perhaps the best characterized centrosomal defects in cancer. However, assessing centrosome numbers is not always an easy task. Most studies rely on counting PCM foci to quantify centrosome number, which is not the most adequate as PCM fragmentation can lead to an increase of acentriolar foci structures, or clustering of extra centrosomes may mask centrosome amplification, reducing them to one PCM foci. Using electron microscopy, an accurate method to quantify centrosome number ${ }^{15}$, would be too laborious for large numbers of cells, as serial sections would be needed to determine precisely the degree of centrosome amplification. The development of systematic approaches to quantify centrosome number would be a significant advance and might prove valuable as a biomarker and prognostic factor for many human cancers ${ }^{17-19}$.

\section{Regulators of centrosome number}

The observation that centrosome amplification occurs in human tumors raised the question of how extra centrosomes arise. Although there are several routes that lead to centrosome amplification, it is still unclear which mechanisms are more prevalent in cancer cells ${ }^{17,21}$. Centrosome overduplication in S-phase induces the formation of multiple centrioles, for example through overexpression of regulators of centrosome duplication (e.g. PLK4/SAK) (Fig. 2A) or mutations in oncogenes or tumor suppressors (e.g. p53, BRCA1) ${ }^{1,14}$. Additionally, prolonged G2 cell cycle arrest can also increase centrosome number ${ }^{22,} 23$. Centrosome amplification can be achieved through cell division failure (e.g. mitotic slippage and cytokinesis failure) and cell-cell fusion, which would also induce tetraploidization ${ }^{24}$. Intriguingly, tumors are composed of heterogeneous populations of cells with normal and increased centrosome number. Thus, how this balance is achieved and how it affects the tumor are interesting open questions.

\section{Centrosomes and cancer: cause, consequence or both?}

The observation that centrosome abnormalities can occur early in pre-malignant lesions and that these defects are extensively correlated with aneuploidy, supports a direct role for extra centrosomes in tumorigenesis 25,26 . Although to date it is still unclear whether centrosome defects are a byproduct of mitotic abnormalities or if they actively contribute to tumorigenesis, significant progress has been made in the field.

Recently, a mechanistic link between extra centrosomes and aneuploidy was established ${ }^{27,28}$. The process of clustering multiple centrosomes at two poles during mitosis, which allows cells to undergo a bipolar division, promotes the formation of chromosome attachment errors, called merotely, which are not sensed by the spindle assembly checkpoint and thus lead to lagging chromosomes and whole chromosome 
aneuploidy 27,28 (Fig. 3A). These studies have established a causal role for centrosome amplification in the generation of chromosome instability, two of the most prominent features of human tumors. Although in primary cells chromosome instability seems to be detrimental, it can promote tumorigenesis in several mouse models ${ }^{29}$. While the mechanism is uncertain, the idea that chromosome instability provides an evolutionary platform important for cancer progression is certainly an exciting possibility ${ }^{29}$. Thus, it is tempting to speculate that the chromosome instability generated by extra centrosomes can contribute to tumorigenesis.

It is important to acknowledge that centrosomes and centrioles have many functions, including their role in polarity and cell migration, and their behavior as signaling platforms in cell cycle transitions and checkpoints ${ }^{30,31}$, all processes affected in tumorigenesis. Moreover, centrosome amplification can lead to the formation of extra cilia, which could potentially modulate signaling output (Fig. 3B). Consistently, the modulation of cilia assembly has been suggested to play an important role in pancreatic cancer potentially through changes in Hh signaling ${ }^{32}$. Hence, alternative roles for centrosome amplification in cancer should not be ignored.

Work in Drosophila using neuroblasts injection has shown that centrosome abnormalities can also promote tumorigenesis by a mechanism that is likely independent of aneuploidy 33,34 (Fig. 3c). In this system, extra centrosomes caused overproliferation of neuroblasts and tumorigenesis due to defects in asymmetric cell division where only minor aneuploidy was detected ${ }^{33}$. Mutations in several centrosomal genes also led to tumorigenesis whereas inducing genomic instability independent of centrosome amplification did not ${ }^{34}$. This suggests that the role of centrosome abnormalities in cancer may ultimately depend on the cellular context from which cancer cells are originated.

\section{Targeting centrosome amplification as a putative therapeutic strategy}

The initial idea that supernumerary centrosomes lead to multipolar cell divisions and aneuploidy does not account for the fact that the gross aneuploidy resulting from multipolar mitoses is likely detrimental to cancer cells ${ }^{35}$. While still a topic of intense discussion, several studies suggests that high levels of aneuploidy could have a tumor suppressor effect at least in certain cellular contexts ${ }^{29}$. Moreover, the progeny of cancer cells that undergo multipolar division are unviable, lending support to the idea that multipolar divisions are a dead end for cancer cells ${ }^{27}$. Thus, it is not surprising that cells have mechanisms that limit the detrimental effects of centrosome amplification, such as centrosome inactivation, centrosome loss and centrosome clustering ${ }^{21}$. Of these, the clustering of extra centrosomes during mitosis which suppresses multipolar divisions, is the best described and has been observed in a variety of tumors (Fig. 3A) ${ }^{33,36-40}$. Thus cells with extra centrosomes might have unique requirements for their survival. Indeed, recent work has shown that centrosome clustering is essential for the survival of cells with supernumerary centrosomes $33,36,38,39$. Furthermore, inhibition of proteins that are essential for centrosome clustering, such as the minus-end directed motor HSET/KIFC1 or the integrin-linked kinase (ILK), selectively kills cancer cells with extra centrosomes, while sparing cells with normal centrosome numbers ${ }^{36,39}$. While inhibitors that target centrosome clustering still require further in vivo validation, these observations open a new possible venue for the development of selective cancer therapeutic strategies.

\section{Centrosomes and diseases of brain development}

Several diseases of brain development have been linked to centrosome and MT-regulating proteins (reviewed in ${ }^{41}$ ). The three most common phenotypes are: disorders of neural migration (e.g. lissencephaly caused by heterozygous mutations in the microtubule binding 
protein LIS1); generalized disorders of growth where the brain is disproportionately affected (e.g. microcephalic osteodysplastic primordial dwarfism type II due to mutations in the PCM protein pericentrin (PCNT) ${ }^{42,43}$ ); and the primary microcephalies where the brain alone is affected and significantly reduced in size, which we now discuss in more detail.

\section{The primary microcephalies}

The crucial role of centrosome proteins in mammalian neurogenesis was discovered through the study of individuals with "autosomal recessive primary microcephaly" (MCPH) where brain growth is significantly reduced. $\mathrm{MCPH}$ is caused by bi-allelic null mutations in any one of seven genes: MICROCEPHALIN, CDK5RAP2, ASPM, CPAP and STIL (reviewed in $^{44}$ ), and newly reported genes CEP152 and WDR62. These MCPH genes all encode ubiquitously expressed proteins that localize to the centrosome at least in some parts of the cell cycle.

\section{Cellular and developmental mechanisms in primary microcephalies}

The human brain is populated by 16 billion neurons generated from a pseudostratified neuroepithelium within the developing brain. The genes affected in MCPH can be subdivided in two classes according to their function: genes involved in centriole duplication and genes involved in centrosome maturation and spindle pole formation. Three MCPH proteins play an important role in centriole duplication, CPAP, STIL and CEP152 (Fig. 2A). CEP152 acts as a scaffold protein to recruit the PLK4 kinase, a trigger of centriole biogenesis, and CPAP, a regulator of centriole-microtubule recruitment and elongation ${ }^{45-47}$ (Fig. 2A). Besides CPAP and CEP152 that play also a role in centrosome maturation, two additional MCPH proteins are required for this process: CDK5RAP2 and ASPM (Fig. 2A). CDK5RAP2 tethers the centrosome and spindle pole during mitosis, which is essential to maintain centriole cohesion ${ }^{48-50}$. In its absence, centrioles detach from the PCM and, as consequence, from the spindle. The orthologue of ASPM has also been shown to be important for spindle pole focusing in Drosophila, and its knockdown also leads to centrosome detachment ${ }^{51}$. Mutations in the gene WDR62 have also been associated with $\mathrm{MCPH}$, although in this case mutations are predominantly missense ${ }^{52-54}$. Nonsense mutations give rise to severe mental retardation and defective neuronal migration 53,54 . WDR62 accumulates at the spindle poles in neural precursors undergoing mitosis, mirroring the localization of ASPM, suggesting a role in centrosome maturation.

Many of the MCPH proteins have been shown to play a role in spindle positioning suggesting that this process is critical for disease development ${ }^{55-58}$. Vertebrate neurogenesis starts with an expansion phase, where symmetric divisions generate neural precursors. This is followed by a phase of differentiation, where neuronal precursors divide asymmetrically to give rise to neurons or basal progenitors. A decrease in the pool of neural precursors is thought to be a cause of MCPH. Although the mechanism is still unclear, one possibility is that proper centrosome functioning might be essential to ensure that divisions are symmetric during the expansion phase and defects in this process would lead to a decrease in neural precursors and MCPH. However, it is puzzling that the MCPH centrosome proteins participate in mitosis in most cell types, yet their mutation results in a specific reduction in brain size. An emerging theme that might help solving that paradox is that the severity of the mutations could lead to different phenotypes, with hypomorphic mutations in CPAP and CEP152 leading to MCPH, but functional null mutations causing a reduction in whole body, as well as the brain ${ }^{59,60}$. It is possible that centrosome structure and function might be affected differently in different tissues or that different tissues compensate differently for the lack of centrosome function during development. It will be very important in the future to correlate each mutation to cellular and developmental 
phenotypes to understand how different tissues might be affected. Animal models of the human mutations should also play an important role in solving this puzzle.

From the clinical perspective, it is now possible to expect that all genetic (mutations) and genomic (copy number variants) alterations leading to primary microcephaly will be diagnosable in the near future. In addition, $\mathrm{MCPH}$ genes may turn out to be valuable markers and tools to manipulate human neural stem cells, which could potentially be used to treat many neurodegenerative disorders.

\section{Centrosomes, cilia and ciliopathies}

Diseases of motile cilia have been known since ciliary assembly defects were associated with Kartagener syndrome, characterized by bronchitis and sinusitis that result from defects in mucus-clearing cilia and often accompanied by sperm immotility ${ }^{61}$. Pathologies caused by defects in motile cilia are referred to as primary ciliary dyskinesia (PCD), which are rare. Ciliary ultrastructural defects are identified in approximately $90 \%$ of PCD patients ${ }^{61}$. Changes in body symmetry, manifested in approximately $50 \%$ of patients with PCD, have shown that ciliary motility is essential to create directional flow in the early embryo initiating the normal left-right developmental program ${ }^{62}$.

In recent years, positional cloning of several other single-gene disorders led to the inclusion of a variety of syndromes in the "ciliopathies list", where mutations lead to abnormal ciliary structure and function and/or the gene product is located at the primary cilia and/or centrosome ${ }^{63}$. This is the case of several rare disorders such as polycystic kidney disease, nephronophthisis, retinitis pigmentosa, Bardet-Biedl, Joubert and Meckel syndromes. Many of these disorders are clinically perplexing as they affect multiple organs including the kidney, retina, brain, bones, liver, and cause kidney failure, blindness, liver fibrosis, obesity or diabetes. The study of the cellular and developmental function of those proteins is contributing to a better understanding of the genesis of those diseases and the function of immotile cilia. In particular in several of those diseases the microtubule-based structure of the cilia is not altered, while its sensory function might be. Recent evidence suggests that this multitude of phenotypes arises from suboptimal ciliary signaling ${ }^{64}$, as discussed below.

Autosomal dominant polycystic kidney disease (ADPKD) is the most frequent lethal dominant disease in the United States and Europe, caused by mutations in the genes $P K D-1$ and $P K D$-2, which code for the proteins polycystin-1 (PC1) and polycystin-1 (PC2) respectively. The localization of PC1 and PC2 in cilia ${ }^{6566}$ (Fig. 2B) and the identification of ciliary defects as a cause for renal cystic phenotypes in the mouse model $\mathrm{Tg} 737$ suggested a role for primary cilia in the pathogenesis of renal cystic disease ${ }^{67}$. Studies of conditional knockout mouse models for PKD-1 ${ }^{68}$ have demonstrated a central role of PC1 in renal tubular morphogenesis as well as tissue maintenance and repair ${ }^{69}$. The product of the gene PKHDI (polycystic kidney and hepatic disease 1) which is mutated in autosomal recessive polycystic kidney disease (ARPKD) ${ }^{70,71}$, also localizes to primary cilia ${ }^{72}$.

Nephronophthisis (NPHP), a recessive kidney disease, which leads to renal fibrosis and corticomedullary cysts, is the most frequent genetic cause for end stage renal failure in the first three decades of life ${ }^{73}$. Mutations in 11 different recessive genes, the Nephrocystins, (NPHP1-NPHP11) have been identified (reviewed in 63,73). NPHP is often associated with other organs besides the kidney. Association with retinal degeneration, known as SeniorLoken syndrome (SLSN), is always seen in cases with mutations of nephrocystin-5 $(N P H P 5)^{74}$. Interestingly, the protein NPHP5 interacts with the retinitis pigmentosa GTPase regulator (RPGR) which is mutated in X-linked retinitis pigmentosa and present in the connecting cilia of photoreceptors ${ }^{74,75}$, The association between both proteins might explain how mutations in NPHP5 lead to retinal phenotypes. 
Meckel-Gruber syndrome (MKS) is an autosomal recessive disease that leads to perinatal death due to dysplasia and malformation in multiple organs. It now appears that multiple recessive mutations in many different genes causing this disease may lead to a wide spectrum of organ involvement. The phenotype depends on the severity of the mutated allele involved. Bardet-Biedl syndrome (BBS) is another disorder that involves many organs. Twelve BBS-associated genes (BBSI-BBS12) have been identified so far and many associate in a complex that sorts membrane proteins, including receptors, to primary cilia ${ }^{76}$, suggesting that defective signaling is at the origin of the disease (Fig. 2B). In the case of Orofaciodigital syndrome type 1 (OFD1) and Jeune's asphyxiating thoracic dystrophy, the defective proteins OFD1 and IFT80 are involved in basal body and cilia formation (Fig. 2B) $(63,73,77)$.

Broad genetic locus heterogeneity has posed a problem for molecular genetic diagnostics of ciliopathies in the past. However, the availability of high-throughput sequencing techniques now offers to individuals with ciliopathies rapid screening for all known ciliopathy-causing genes. This approach, in conjunction with whole exome and genome sequencing will facilitate detection of the remaining unknown ciliopathy genes ${ }^{77}$. Therapeutic options in degenerative ciliopathies are being developed in animal models. In cystic kidneys diseases therapeutic trials are ongoing studying the effects of rapamycin, octreotide, and a vasopressin receptor antagonist. The time interval of almost 10 years between onset of first renal symptoms and terminal renal failure offers a theoretical time window for treatment or prevention. Knowledge of an individual's specific mutation will permit classification of particular genetic subsets of patients for specific future therapeutic trials.

\section{Cellular and developmental mechanisms in ciliopathies}

Genes that are mutated in ciliopathies, as discussed above, can affect ciliary signaling in different ways: through changes in cilia structure, in targeting signaling molecules appropriately, or at the level of the sensory/signaling molecules (Fig. 2B). Changes in the Hh pathway are associated with several developmental defects, including polydactily, neural tube and craniofacial defects. Mouse mutants deficient in specific genes involved in intraflagellar transport (IFT) including Kif3a exhibit Hedgehog mutant phenotypes with cystic kidneys (reviewed in ${ }^{10}$ ). Mutations in the NPHP7/Glis2 gene, were identified as the cause of NPHP type $7^{78}$. Glis 2 mutant mice showed severe renal atrophy and fibrosis resembling human nephronophthisis ${ }^{78}$. How this cystogenic phenotype arises is still not clear, but it has been suggested to be mediated by a Hh role in epithelial to mesenchymal transition $^{79}$. Several molecules, which have been associated with cilia-related diseases, play a role in the Wnt pathway. For instance, Inversin/NPHP2 function is necessary to switch from the canonical to the non-canonical Wnt/planar cell polarity (PCP) pathway (Fig. 2B). Moreover, several BBS proteins cooperate with non-canonical Wnt signaling in $\mathrm{PCP}^{80,81}$. There is aberrant orientation of the mitotic spindle in mouse or rat models with mutation of the cystic kidney disease genes $H n f 1^{82}, P k h d 1^{82}$, and Kif $3 a^{83}$ suggesting that defective spindle orientation could contribute to kidney cysts. However, this model has not been confirmed for $\mathrm{PKD}^{84,85}$, where loss of oriented cell division is neither sufficient to produce kidney cysts nor required to initiate cyst formation. The role of proliferation in the genesis of ciliopathies phenotypes is supported by the fact that the cyclin-dependent kinase inhibitor roscovitine can efficiently treat two renal cystic mouse models $(j c k \text { and } c p k)^{86}$. It is not clear whether all ciliopathies phenotypes result from dysfunctional ciliary signaling, or whether in some cases dysfunctional centrosome signaling and/or cytoskeleton disorganization alone trigger the disease. 


\section{Dysplasia versus degeneration in ciliopathies}

Very recently the surprising discovery was made that mutation of the same recessive gene (this includes NPHP3, NPHP6 or NPHP8) may cause very different NPHP-like ciliopathy phenotypes. In these genes, the presence of 2 null alleles causes the severe, early-onset phenotype of perinatal lethal Meckel syndrome with dysplasia/malformation in most organs, whereas individuals with hypomorphic (missense) alleles are normal at birth and over years develop degenerative phenotypes of nephronophthisis, retinal degeneration and liver fibrosis ${ }^{87-90}$. It appears that null mutation already manifests during organ development resulting in dysplasia, whereas, hypomorphic mutations manifest in adult tissue during tissue maintenance and repair. A similar effect was described for $P k d l$, where the occurrence of cysts in the kidney depends on developmental status ${ }^{68,83}$. These data suggest that tissues have very different requirements for the function of those genes in development and homeostasis.

\section{Concluding remarks and future perspectives}

In this review, we highlighted the role of centrosomes, centrioles and cilia in a broad spectrum of genetic and acquired diseases. The recent identification of genes involved in ciliopathies and diseases of brain development helps to clarify how organelle biogenesis and function are targeted, as well as their developmental implications. In primary microcephalies, proteins participating in the centrosome cycle are affected. It has been suggested that the most plausible explanation for the observed small brain is the deregulation of the balance between symmetric and asymmetric divisions of neural progenitors, which normally guarantees genesis of the right cell type, at the right time. In primary cilia dyskinesia, cilia structure and motility are affected with clear implications in processes that involve cilia motility. Finally, in the majority of ciliopathies, there is either disruption of primary cilia assembly or of the transport of signalling molecules to centrosomes and cilia, suggesting overall problems in signalling that affect cell proliferation, polarity and/or orientation of cell division.

While certain diseases of centrosomes and cilia may be manifested in the same tissues and share phenotypes, others are dramatically different and diverse. Mutations in the same gene may lead to strongly different phenotypes. Moreover, microcephaly only rarely and inconsistently occurs in other disease phenotypes caused by bi-allelic mutation of centrosomal proteins, e.g. Joubert, Bardet-Biedl, Meckel syndromes. And the corollary holds true, that the extra-cerebral features of these diseases do not occur in MCPH. Strikingly, both molecules as well as the tissues that are affected in each disease are different. Overall these studies highlight that developmental and homeostasis pathways vary between tissues and developmental stages, leading to different sensitivity to the failure of particular pathways and gene interactions. In particular it is very unlikely that the mutations observed in microcephaly completely disrupt centriole formation in all tissues, as this would generally impair cilia formation ${ }^{1}$, leading to ciliopathies.

Despite significant progress made in the field of centrosomes and cancer, the role of centrosomal abnormalities in human tumorigenesis still remains uncertain. Several mouse models that target centrosomal proteins can form tumors. However, in these models, the observed centrosome defects are usually accompanied by increase in ploidy ${ }^{91-94}$. Thus, the development of new models that can disentangle the increase in ploidy from the centrosomal defects is crucial to fully address this question. It will be also important to understand the consequences of centrosome abnormalities in situ, in particular whether some of the defects observed in the genetic diseases, such as abnormal cell proliferation and centrosome/ciliary signaling, are also observed. 
An interesting lesson to learn from the genetic diseases discussed here is that the requirements for centrosome and cilia normal function are likely to be tissue-specific. Perhaps different centrosome and cilia functions might also be targeted in different types of cancer, depending on tissue type and time of appearance of their abnormalities. Regardless of substantial advances, we are only starting to unveil the role of centrosomes and cilia in human diseases and many exciting questions still lie ahead in the field.

\section{Acknowledgments}

We would like to thank Zita Carvalho-Santos and Ofélia Carvalho for help with figures and Gaelle Marteil for discussing the manuscript. Research in MBD lab is funded by Fundação para a Ciência e Tecnologia, Fundação Calouste Gulbenkian, European Molecular Biology Organization and the European Research Council. SG is funded with a postdoctoral fellowship from the Harvard-Portugal programme (FCT) for a collaborative grant between the MBD and the DP laboratories. FH is an Investigator of the Howard Hughes Medical Institute, the Frederick G. L. Huetwell Professor, and a Doris Duke Distinguished Clinical Scientist. DP is a Howard Hughes Medical Institute Investigator. We would like to thank Michel Bornens for his permission to use Figure 1A'.

\section{References}

1. Bettencourt-Dias M, Glover DM. Centrosome biogenesis and function: centrosomics brings new understanding. Nat Rev Mol Cell Biol. 2007; 8:451-463. [PubMed: 17505520]

2. Yamashita YM, et al. Asymmetric inheritance of mother versus daughter centrosome in stem cell division. Science. 2007; 315:518-521. [PubMed: 17255513]

3. Conduit PT, Raff JW. Cnn dynamics drive centrosome size asymmetry to ensure daughter centriole retention in drosophila neuroblasts. Curr Biol. 2010; 20:2187-2192. [PubMed: 21145745]

4. Januschke J, et al. Drosophila neuroblasts retain the daughter centrosome. Nat Commun. 2:243. [PubMed: 21407209]

5. Wang X, et al. Asymmetric centrosome inheritance maintains neural progenitors in the neocortex. Nature. 2009; 461:947-955. [PubMed: 19829375]

6. Cunha-Ferreira I, et al. From zero to many: control of centriole number in development and disease. Traffic. 2009; 10:482-498. [PubMed: 19416494]

7. Debec A, et al. Centrioles: active players or passengers during mitosis? Cell Mol Life Sci. 2010; 67:2173-2194. [PubMed: 20300952]

8. Rodrigues-Martins A, et al. From centriole biogenesis to cellular function: Centrioles are essential for cell division at critical developmental stages. Cell Cycle. 2008; 7:11-16. [PubMed: 18196975]

9. Nachury MV, et al. Trafficking to the ciliary membrane: how to get across the periciliary diffusion barrier? Annu Rev Cell Dev Biol. 2010; 26:59-87. [PubMed: 19575670]

10. Goetz SC, Anderson KV. The primary cilium: a signalling centre during vertebrate development. Nat Rev Genet. 2010; 11:331-344. [PubMed: 20395968]

11. Gerdes JM, et al. The vertebrate primary cilium in development, homeostasis, and disease. Cell. 2009; 137:32-45. [PubMed: 19345185]

12. Wallingford JB, Mitchell B. Strange as it may seem: the many links between Wnt signaling, planar cell polarity, and cilia. Genes Dev. 2011; 25:201-213. [PubMed: 21289065]

13. Boveri T. Concerning the Origin of Malignant Tumours by Theodor Boveri (1914). Translated and annotated by Henry Harris. Journal Cell Science. 2008; 121:1-84.

14. Fukasawa K. Oncogenes and tumour suppressors take on centrosomes. Nat Rev Cancer. 2007; 7:911-924. [PubMed: 18004399]

15. Lingle WL, et al. Centrosome hypertrophy in human breast tumors: implications for genomic stability and cell polarity. Proc Natl Acad Sci U S A. 1998; 95:2950-2955. [PubMed: 9501196]

16. Pihan GA, et al. Centrosome defects and genetic instability in malignant tumors. Cancer Res. 1998; 58:3974-3985. [PubMed: 9731511]

17. Nigg EA. Centrosome aberrations: cause or consequence of cancer progression? Nat Rev Cancer. 2002; 2:815-825. [PubMed: 12415252] 
18. Kramer A, et al. Centrosome aberrations in hematological malignancies. Cell Biol Int. 2005; 29:376-384.

19. Zyss D, Gergely F. Centrosome function in cancer: guilty or innocent? Trends Cell Biol. 2009; 19:334-346. [PubMed: 19570677]

20. Nigg EA. Origins and consequences of centrosome aberrations in human cancers. Int J Cancer. 2006; 119:2717-2723. [PubMed: 17016823]

21. Godinho SA, et al. Centrosomes and cancer: how cancer cells divide with too many centrosomes. Cancer Metastasis Rev. 2009; 28:85-98. [PubMed: 19156503]

22. Loncarek J, et al. Centriole reduplication during prolonged interphase requires procentriole maturation governed by Plk1. Curr Biol. 2010; 20:1277-1282. [PubMed: 20656208]

23. Bourke E, et al. DNA damage induces Chk1-dependent centrosome amplification. EMBO Rep. 2007; 8:603-609. [PubMed: 17468739]

24. Ganem NJ, et al. Tetraploidy, aneuploidy and cancer. Curr Opin Genet Dev. 2007; 17:157-162. [PubMed: 17324569]

25. Lingle WL, et al. Centrosome amplification drives chromosomal instability in breast tumor development. Proc Natl Acad Sci U S A. 2002; 99:1978-1983. [PubMed: 11830638]

26. Pihan GA, et al. Centrosome abnormalities and chromosome instability occur together in preinvasive carcinomas. Cancer Res. 2003; 63:1398-1404. [PubMed: 12649205]

27. Ganem NJ, et al. A mechanism linking extra centrosomes to chromosomal instability. Nature. 2009; 460:278-282. [PubMed: 19506557]

28. Silkworth WT, et al. Multipolar spindle pole coalescence is a major source of kinetochore misattachment and chromosome mis-segregation in cancer cells. PLoS One. 2009; 4:e6564. [PubMed: 19668340]

29. Schvartzman JM, et al. Mitotic chromosomal instability and cancer: mouse modelling of the human disease. Nat Rev Cancer. 2010; 10:102-115. [PubMed: 20094045]

30. Sluder G. Two-way traffic: centrosomes and the cell cycle. Nat Rev Mol Cell Biol. 2005; 6:743748. [PubMed: 16231423]

31. Doxsey S, et al. Centrosomes in Cellular Regulation. Annu Rev Cell Dev Biol. 2004

32. Seeley ES, et al. Pancreatic cancer and precursor pancreatic intraepithelial neoplasia lesions are devoid of primary cilia. Cancer Res. 2009; 69:422-430. [PubMed: 19147554]

33. Basto R, et al. Centrosome amplification can initiate tumorigenesis in flies. Cell. 2008; 133:10321042. [PubMed: 18555779]

34. Castellanos E, et al. Centrosome Dysfunction in Drosophila Neural Stem Cells Causes Tumors that Are Not due to Genome Instability. Curr Biol. 2008

35. Brinkley BR. Managing the centrosome numbers game: from chaos to stability in cancer cell division. Trends Cell Biol. 2001; 11:18-21. [PubMed: 11146294]

36. Kwon M, et al. Mechanisms to suppress multipolar divisions in cancer cells with extra centrosomes. Genes Dev. 2008

37. Quintyne NJ, et al. Spindle multipolarity is prevented by centrosomal clustering. Science. 2005; 307:127-129. [PubMed: 15637283]

38. Leber B, et al. Proteins required for centrosome clustering in cancer cells. Sci Transl Med. 2010; 2:33ra38.

39. Fielding AB, et al. A critical role of integrin-linked kinase, ch-TOG and TACC3 in centrosome clustering in cancer cells. Oncogene. 2010; 30:521-534. [PubMed: 20838383]

40. Ring D, et al. Mitosis in a cell with multiple centrioles. J Cell Biol. 1982; 94:549-556. [PubMed: 7130271]

41. Friocourt G, et al. Role of cytoskeletal abnormalities in the neuropathology and pathophysiology of type I lissencephaly. Acta Neuropathol. 121:149-170. [PubMed: 21046408]

42. Rauch A, et al. Mutations in the pericentrin (PCNT) gene cause primordial dwarfism. Science. 2008; 319:816-819. [PubMed: 18174396]

43. Griffith E, et al. Mutations in pericentrin cause Seckel syndrome with defective ATR-dependent DNA damage signaling. Nat Genet. 2008; 40:232-236. [PubMed: 18157127] 
44. Thornton GK, Woods CG. Primary microcephaly: do all roads lead to Rome? Trends Genet. 2009; 25:501-510. [PubMed: 19850369]

45. Dzhindzhev NS, et al. Asterless is a scaffold for the onset of centriole assembly. Nature. 2010; 467:714-718. [PubMed: 20852615]

46. Hatch EM, et al. Cep152 interacts with Plk4 and is required for centriole duplication. J Cell Biol. 2010; 191:721-729. [PubMed: 21059850]

47. Cizmecioglu O, et al. Cep152 acts as a scaffold for recruitment of Plk4 and CPAP to the centrosome. J Cell Biol. 191:731-739. [PubMed: 21059844]

48. Barrera JA, et al. CDK5RAP2 regulates centriole engagement and cohesion in mice. Dev Cell. 2010; 18:913-926. [PubMed: 20627074]

49. Barr AR, et al. CDK5RAP2 functions in centrosome to spindle pole attachment and DNA damage response. J Cell Biol. 2010; 189:23-39. [PubMed: 20368616]

50. Lee S, Rhee K. CEP215 is involved in the dynein-dependent accumulation of pericentriolar matrix proteins for spindle pole formation. Cell Cycle. 2010; 9:774-783. [PubMed: 20139723]

51. Gonzalez C, et al. Mutations at the asp locus of Drosophila lead to multiple free centrosomes in syncytial embryos, but restrict centrosome duplication in larval neuroblasts. J Cell Sci. 1990; 96(Pt 4):605-616. [PubMed: 2283359]

52. Bilguvar K, et al. Whole-exome sequencing identifies recessive WDR62 mutations in severe brain malformations. Nature. 467:207-210. [PubMed: 20729831]

53. Yu TW, et al. Mutations in WDR62, encoding a centrosome-associated protein, cause microcephaly with simplified gyri and abnormal cortical architecture. Nat Genet. 42:1015-1020. [PubMed: 20890278]

54. Nicholas AK, et al. WDR62 is associated with the spindle pole and is mutated in human microcephaly. Nat Genet. 2010; 42:1010-1014. [PubMed: 20890279]

55. Giansanti MG, et al. The role of centrosomes and astral microtubules during asymmetric division of Drosophila neuroblasts. Development. 2001; 128:1137-1145. [PubMed: 11245579]

56. Basto R, et al. Flies without centrioles. Cell. 2006; 125:1375-1386. [PubMed: 16814722]

57. Megraw TL, et al. Zygotic development without functional mitotic centrosomes. Curr Biol. 2001; 11:116-120. [PubMed: 11231128]

58. Fish JL, et al. Aspm specifically maintains symmetric proliferative divisions of neuroepithelial cells. Proc Natl Acad Sci U S A. 2006; 103:10438-10443. [PubMed: 16798874]

59. Al-Dosari MS, et al. Novel CENPJ mutation causes Seckel syndrome. J Med Genet. 2010; 47:411414. [PubMed: 20522431]

60. Kalay E, et al. CEP152 is a genome maintenance protein disrupted in Seckel syndrome. Nat Genet. 2010; 43:23-26. [PubMed: 21131973]

61. Zariwala MA, et al. Genetic defects in ciliary structure and function. Annu Rev Physiol. 2007; 69:423-450. [PubMed: 17059358]

62. Nonaka S, et al. Randomization of left-right asymmetry due to loss of nodal cilia generating leftward flow of extraembryonic fluid in mice lacking KIF3B motor protein. Cell. 1998; 95:829837. [PubMed: 9865700]

63. Hildebrandt F, et al. Ciliopathies. N Engl J Med. 2011; 364:1533-1543. [PubMed: 21506742]

64. Baker K, Beales PL. Making sense of cilia in disease: the human ciliopathies. Am J Med Genet C Semin Med Genet. 2009; 151C:281-295. [PubMed: 19876933]

65. Barr MM, Sternberg PW. A polycystic kidney-disease gene homologue required for male mating behaviour in C. elegans. Nature. 1999; 401:386-389. [PubMed: 10517638]

66. Yoder BK, et al. The polycystic kidney disease proteins, polycystin-1, polycystin-2, polaris, and cystin, are co-localized in renal cilia. J Am Soc Nephrol. 2002; 13:2508-2516. [PubMed: 12239239]

67. Pazour GJ, et al. Chlamydomonas IFT88 and its mouse homologue, polycystic kidney disease gene $\operatorname{tg} 737$, are required for assembly of cilia and flagella. J Cell Biol. 2000; 151:709-718. [PubMed: 11062270]

68. Piontek K, et al. A critical developmental switch defines the kinetics of kidney cyst formation after loss of Pkd1. Nat Med. 2007; 13:1490-1495. [PubMed: 17965720] 
69. Kim E, Walz G. Sensitive cilia set up the kidney. Nat Med. 2007; 13:1409-1411. [PubMed: 18064029]

70. Ward CJ, et al. The gene mutated in autosomal recessive polycystic kidney disease encodes a large, receptor-like protein. Nat Genet. 2002; 30:259-269. [PubMed: 11919560]

71. Onuchic LF, et al. PKHD1, the polycystic kidney and hepatic disease 1 gene, encodes a novel large protein containing multiple immunoglobulin-like plexin-transcription-factor domains and parallel beta-helix 1 repeats. Am J Hum Genet. 2002; 70:1305-1317. [PubMed: 11898128]

72. Kim I, et al. Fibrocystin/polyductin modulates renal tubular formation by regulating polycystin-2 expression and function. J Am Soc Nephrol. 2008; 19:455-468. [PubMed: 18235088]

73. Hildebrandt F, Zhou W. Nephronophthisis-associated ciliopathies. J Am Soc Nephrol. 2007; 18:1855-1871. [PubMed: 17513324]

74. Otto E, Loeys B, Khanna H, Hellemans J, Sudbrak R, Fan S, Muerb U, O'Toole JF, Helou J, Attanasio M, Utsch B, Sayer JA, Lillo C, Jimeno D, Coucke P, De Paepe A, Reinhardt R, Klages S, Tsuda M, Kawakami I, Kusakabe T, Omran H, Imm A, Tippens M, Raymond PA, Hill J, Beales P, He S, Kispert A, Margolis B, Williams DS, Swaroop A, Hildebrandt F. A novel ciliary IQ domain protein, NPHP5, is mutated in Senior-Loken syndrome (nephronophthisis with retinitis pigmentosa), and interacts with RPGR and calmodulin. Nat Genet. 2005; 37:282-288. [PubMed: 15723066]

75. Chang B, et al. In-frame deletion in a novel centrosomal/ciliary protein CEP290/NPHP6 perturbs its interaction with RPGR and results in early-onset retinal degeneration in the rd16 mouse. Hum Mol Genet. 2006; 15:1847-1857. [PubMed: 16632484]

76. Jin $\mathrm{H}$, et al. The conserved Bardet-Biedl syndrome proteins assemble a coat that traffics membrane proteins to cilia. Cell. 2010; 141:1208-1219. [PubMed: 20603001]

77. Otto EA, et al. Candidate exome capture identifies mutation of SDCCAG8 as the cause of a retinalrenal ciliopathy. Nat Genet. 2010; 42:840-850. [PubMed: 20835237]

78. Attanasio M, et al. Loss of GLIS2 causes nephronophthisis in humans and mice by increased apoptosis and fibrosis. Nat Genet. 2007; 39:1018-1024. [PubMed: 17618285]

79. Gascue C, et al. Cystic diseases of the kidney: ciliary dysfunction and cystogenic mechanisms. Pediatr Nephrol. 2011

80. Ross AJ, et al. Disruption of Bardet-Biedl syndrome ciliary proteins perturbs planar cell polarity in vertebrates. Nat Genet. 2005; 37:1135-1140. [PubMed: 16170314]

81. Germino GG. Linking cilia to Wnts. Nat Genet. 2005; 37:455-457. [PubMed: 15858588]

82. Fischer E, et al. Defective planar cell polarity in polycystic kidney disease. Nat Genet. 2006; 38:21-23. [PubMed: 16341222]

83. Patel V, et al. Acute kidney injury and aberrant planar cell polarity induce cyst formation in mice lacking renal cilia. Hum Mol Genet. 2008; 17:1578-1590. [PubMed: 18263895]

84. Nishio S, et al. Loss of oriented cell division does not initiate cyst formation. J Am Soc Nephrol. 2010; 21:295-302. [PubMed: 19959710]

85. Chizhikov VV, et al. Cilia proteins control cerebellar morphogenesis by promoting expansion of the granule progenitor pool. J Neurosci. 2007; 27:9780-9789. [PubMed: 17804638]

86. Bukanov NO, et al. Long-lasting arrest of murine polycystic kidney disease with CDK inhibitor roscovitine. Nature. 2006

87. Bergmann C, et al. Loss of nephrocystin-3 function can cause embryonic lethality, Meckel-Gruberlike syndrome, situs inversus, and renal-hepatic-pancreatic dysplasia. Am J Hum Genet. 2008; 82:959-970. [PubMed: 18371931]

88. Helou J, et al. Mutation analysis of NPHP6/CEP290 in patients with Joubert syndrome and SeniorLoken syndrome. J Med Genet. 2007; 44:657-663. [PubMed: 17617513]

89. Delous M, et al. The ciliary gene RPGRIP1L is mutated in cerebello-oculo-renal syndrome (Joubert syndrome type B) and Meckel syndrome. Nat Genet. 2007; 39:875-881. [PubMed: 17558409]

90. Otto EA, et al. Hypomorphic mutations in meckelin (MKS3/TMEM67) cause nephronophthisis with liver fibrosis (NPHP11). J Med Genet. 2009; 46:663-670. [PubMed: 19508969] 
91. Shao S, et al. Centrosomal Nlp is an oncogenic protein that is gene-amplified in human tumors and causes spontaneous tumorigenesis in transgenic mice. J Clin Invest. 2010; 120:498-507. [PubMed: 20093778]

92. Wang X, et al. Overexpression of aurora kinase A in mouse mammary epithelium induces genetic instability preceding mammary tumor formation. Oncogene. 2006; 25:7148-7158. [PubMed: 16715125]

93. Grisendi S, et al. Role of nucleophosmin in embryonic development and tumorigenesis. Nature. 2005

94. Rosario CO, et al. Plk4 is required for cytokinesis and maintenance of chromosomal stability. Proc Natl Acad Sci U S A. 2010; 107:6888-6893. [PubMed: 20348415]

95. Paintrand M, et al. Centrosome organization and centriole architecture: their sensitivity to divalent cations. J Struct Biol. 1992; 108:107-128. [PubMed: 1486002]

96. Nigg EA, Raff JW. Centrioles, centrosomes, and cilia in health and disease. Cell. 2009; 139:663678. [PubMed: 19914163] 


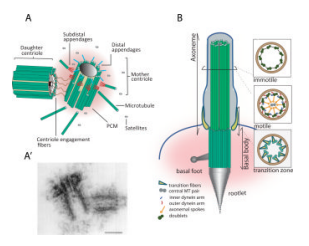

Figure 1. Centrosome and cilia structure

The centriole is a structural constituent of centrosomes, cilia and flagella. (A) The canonical centriole has nine MT triplets and is $\sim 0.5 \mu \mathrm{m}$ long and $0.2 \mu \mathrm{m}$ in diameter. Each centrosome is composed by a mother and daugther centriole present in an orthogonal configuration and surrounded by a matrix of proteins called the pericentriolar material (PCM). The older centriole (mother) shows subdistal appendages, where MTs are docked, and distal appendages, which are important for docking to the plasma membrane. Satellites are granular structures surrounding the centrosome that are implicated in trafficking of material involved in centriole assembly. (A') Electron micrograph of a centrosome; scale bar: $0.2 \mu \mathrm{m}$ (reproduced with permission from ${ }^{95}$ ). (B) In many cells the centriole, then called basal body, migrates and tethers to the plasma membrane via its appendages and seeds the growth of cilia and flagella. The skeleton of cilia and flagella, called the axoneme, results from a continuation of the basal body structure and might be composed of nine doublets with no dynein arms nor central pair, as it is in the case of most immotile cilia; or nine MT doublets with dynein arms and a central MT pair, as it is for most motile cilia. The distal part of the basal body is called transition zone, where the outer tubule stops growing. During centriole to basal body differentiation the acquisition of specialized structures such as striated rootlets, basal feet and transitional fibres, will provide mechanical support to cilia, anchor the basal body to the apical cytoskeleton and serve as platforms for the docking of ciliary components, respectively ${ }^{11}$. 


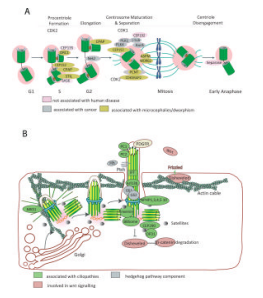

Figure 2. Centrosome and cilia biogenesis and human disease

A. Centriole biogenesis. PLK4 triggers centriole biogenesis. It is recruited to the centrosome by CEP152. CEP152 also binds other molecules essential to this process, such as CPAP (also called SAS4 in Caenohabditis elegans). Procentriole formation begins in S phase upon recruitment of SAS6, CEP135 and STIL (also called SAS5), which are needed to form the cartwheel, a structure that helps in defining the centriole nine-fold symmetry. CPAP also plays a role in centriole elongation (for a review see ${ }^{1,96}$ ). CDK2 activity may be necessary for speeding up procentriole formation and elongation, hence coordinating this event with DNA replication. In $\mathrm{G} 2$, the daughter centriole reaches full elongation and maturation with the recruitment of several molecules that are needed for microtubule nucleation, stability and focusing to the pericentriolar material (PCM), including pericentrin (PCTN), CEP192 (also called SPD2 in C. elegans), CDK5RAP2 (also called CNN in Drosophila) and ASPM. CDK1 activity increases in G2, regulating a variety of molecules and processes needed for entry into mitosis, such as changes in microtubule dynamics. Through the concerted action of molecules such as the kinase Nek2, the two centrosomes separate. When a cell exits mitosis, the centrioles within the centrosome disengage through the action of PLK1 and separase. That process may allow recruitment or activation of molecules necessary for duplication and ensures that daughter centrioles can only form after this point, preventing reduplication. Molecules involved in preventing DNA rereplication, such as ORC1 have a similar role in preventing centriole reduplication (for a review see ${ }^{1,96}$ ). B. Cilia assembly/ function and human disease. Mother centriole appendices dock to a vesicle, after which axoneme growth starts, followed by fusion of the vesicle to the plasma membrane. Migration of the centriole is dependent on the actin cytoskeleton and molecules such as MKS1, which is mutated in Meckel-Grueber syndrome. Several signalling pathways operate in ciliated cells, some of which are dependent on the presence of the cilium. Calcium signalling operates through membrane receptors and calcium channels on the ciliary membrane such as polycystin 1 and 2 respectively (PC1 and PC2). Hedgehog (Hh) signalling operates through the cilium in vertebrates: upon binding of Hh to its receptor Patched (Ptch1) a cascade of events starts within cilia and the body of the cell leading to the expression of target genes (see text; for a review see ${ }^{10}$ ). Wnt signalling is modulated through several components that localise at the centrosome and cilia. Several players in human disease, such as inversin (also called NPHP2) and the BBS proteins, which are part of the BBsome and are involved in protein trafficking, have been shown to play an important role in the switch between canonical and non-canonical Wnt pathways, through the regulation of $\beta$-catenin degradation, hence potentially regulating polarity, spindle positioning and proliferation (for a review see 11,12 ). The centriolar satellites may play an important role in human disease as several ciliopathy proteins, such as BBS4, CEP290 (also called NPHP6) and OFD1 have been shown to localise both to centrioles and satellites. Several proteins mutated in human disease, such as the NPHP1,3,4,5 and 10 localise to the transition zone. IFT components, which are involved in transporting molecules in and out of the cilia, including molecules involved in cilia assembly are also mutated in ciliopathies (e.g. IFT80, Dync2h1). 


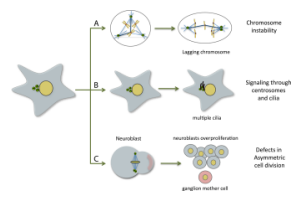

Figure 3. Centrosome and cancer

Possible mechanisms of how extra centrosomes could affect tumorigenesis. (A) Extra centrosomes can directly induce aneuploidy by forcing cells to undergo a multipolar intermediate during spindle assembly that lead to an increase of merotelic chromosome attachments and lagging chromosomes during mitosis. (B) Centrosome amplification can affect signalling, for example by modulating cilia number. (C) The presence of extra centrosomes can cause defects in asymmetric cell division in Drosophila neuroblasts, leading to an overproliferation of the stem cell population. 